\title{
Defect Structures in CuZr Martensite, Studies by CTEM and HRTEM
}

\author{
J.W. Seo and D. Schryvers \\ University of Antwerp, RUCA, EMAT, Groenenborgerlaan 171, 2020 Antwerp, Belgium
}

\begin{abstract}
Defect structures in CuZr martensite are studied by conventional and high-resolution transmission electron microscopy. Two different types of planar defects are observed. It is concluded that both defect types can be inherited from anti-phase boundaries in the austenite phase. With respect to the martensitic transformation, both defect types can be explained by the different shuffles of atoms after or during the distortion to the monoclinic angle.
\end{abstract}

\section{INTRODUCTION}

The CuZr system has for the equiatomic composition a stable $\mathrm{B} 2$ phase with a $\mathrm{CsCl}$ type bcc structure which exists at temperatures above $715^{\circ} \mathrm{C}$ [1]. By a rapid cooling of this high-temperature line phase to below $140^{\circ} \mathrm{C}$ the decomposition to the neighbouring stable phases is suppressed and it transforms into two metastable phases with monoclinic structures [2]. These phases have martensitic characteristics and show shape memory behaviour [3,4] similar to other B2 phase alloys [5].

The structures of the martensitic phases were recently investigated by X-ray diffractometry and transmission electron microscopy (TEM) $[3,6,7,8]$. Based on these results one small and one large unit cell were proposed and their possible atomic positions suggested [6,7]. For the small structure an evidence for the B19' NiTi-type unit cell was found while the large unit cell was considered as a superstructure of the small cell with a doubling along the a- and b-axis. An extensive TEM study on the microstructure and morphology of these two monoclinic phases has been reported [7,8]. A typical plate like structure with internal microtwinning was observed, the latter occurring preferentially in one of both phases $[3,8]$.

In the present paper the results of an extensive conventional (CTEM) and high-resolution (HRTEM) transmission electron microscopy investigation of the defect structures will be presented. The different defect structures will be analysed and discussed with respect to the martensitic transformation.

\section{EXPERIMENTAL PROCEDURE}

$\mathrm{CuZr}$ alloys are arc-melted using electrolytic $\mathrm{Cu}$ and iodide $\mathrm{Zr}$ under pregettered $\mathrm{Ar}$ atmosphere. As received materials as well as homogenised for several hours at $800^{\circ} \mathrm{C}$ were investigated. These samples are prepared by J.V. Humbeeck at Dept. MTM, K.U. Leuven, Belgium, and G.S. Firstov at Inst. Metal Physics in Kiev, Ukraine. For TEM investigation they were bored ultrasonically to $3 \mathrm{~mm}$ diameter and grinded to a final thickness of about $120 \mathrm{~nm}$. The samples were thinned by electropolishing in a Tenupol 3 twin jet polisher using a solution of $9 \%$ nitric acid in methanol/butoxyethanol of ratio $2: 1$ at $-30^{\circ} \mathrm{C}$ and a voltage of about $30 \mathrm{~V}$ with a current around $0.1 \mathrm{~A}$. The CTEM studies were performed in a Philips CM20 microscope equipped with a $\mathrm{LaB}_{6}$ filament and a twin lens. For HRTEM a JEOL 4000EX microscope was used operating by $400 \mathrm{kV}$ with a point resolution of about $0.17 \mathrm{~nm}$.

\section{RESULTS}

The most predominant defects in CuZr martensite are twins. A detailed study on the microstructure of the micro as well as macrotwins has been presented in ref. [3,8,9].

Other planar defects are frequently observed in CuZr martensite $[8,9]$. As can be seen in the other contribution of this conference [9] most of these defects indicate straight segments which are nearly 
perpendicular to microtwins. Others show a habit plane with an inclined angle of $21^{\circ}$ to the microtwin plane or a habit plane which is not well defined.

In fig. 1 a [110] HRTEM image of a defect is shown which was observed in a low magnification image to cross the microtwins by an angle of $21^{\circ}$. Here the defect crosses the image diagonally from the lower-left to the upper-right corner. The width of the defect region is in the range of 1-2 unit cells and indicates an edge-on configuration in this zone. The habit plane of the defect can be found in this scale as not well defined unlike the observation done in low magnification (see contribution [9]). By SAED the superreflections of the superstructure were observed and the presence of the superstructure was concluded. Image simulations which are carried out in this zone using the atomic structure of the superstructure were consistent with the experimental images for several defocus values [8]. Using the resulting imaging code the unit cell of the $\mathrm{CuZr}$ superstructure can be correlated with the indication in the figure. Viewing this lattice image along a grazing incidence parallel with (110) lattice fringes a displacement of $1 / 2$ of the (110) interplanar spacing is found while no displacement of (001) lattice fringes is observed. This type of defects will be referred to the defect type A.

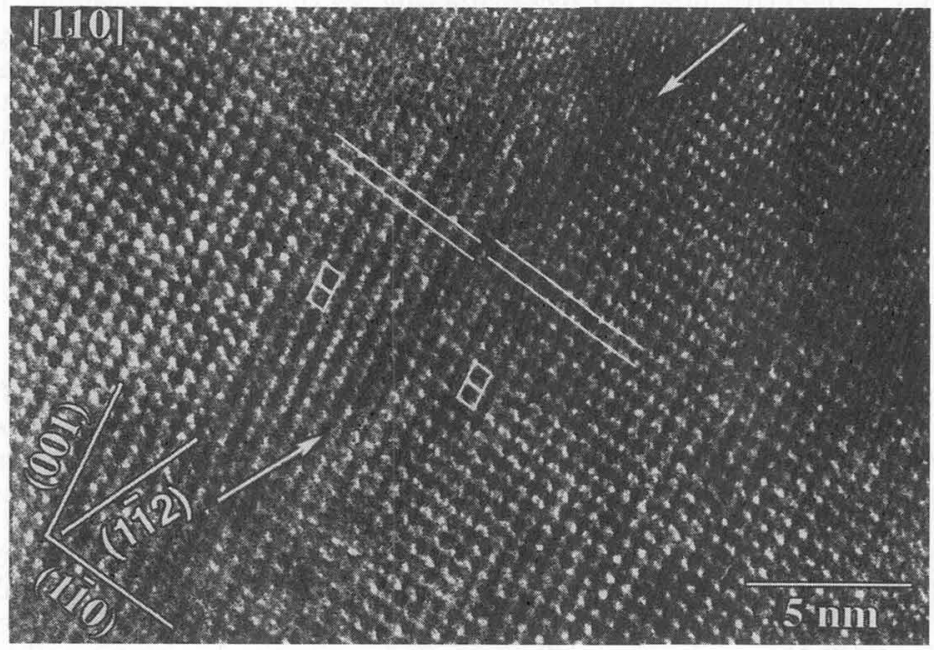

Figure 1: [110] HRTEM image of a defect of type A with a displacement of $1 / 2$ of the (110) lattice fringes. For the image interpretation the white dots can be correlated with the projected unit cell as indicated in the figure.

For the defect nearly perpendicular to the microtwins (see contribution [9]) the habit plane is parallel to the (110) plane of a twin variant and parallel to the (112) plane of the other. In fig. 2 a HRTEM image of a planar defect with a zigzag-like morphology can be seen. Here, some segments are found which are again almost perpendicular to the microtwins, indeed for these regions the habit plane is parallel to the (110) plane of a twin variant. Other segments which are not perpendicular to the microtwins are parallel with higher indexed planes but close to (111) (or (223) of the other variant). In some segments the defect is straight within a given microtwin and changes its orientation by meeting the microtwin boundary. Especially for microtwins with a width of about $10 \mathrm{~nm}$ this correlation can be seen clearly as indicated by white arrows in fig. 2 . The orientation change of the defect is not symmetrical to the mirror plane of microtwins. For the twinned area with a twin width smaller than 2-3 nm the interaction between them is not clear but it can be found that the habit plane is here not well defined especially in a scale of 1-2 unit cells. It is observed that the microtwins frequently end at the defect, the microtwin boundaries are mostly not correlated over the defect.

In fig. 3 a HRTEM image of a selected area in fig. 2 is shown. In order to allow for a useful description a region with the same microtwin variant on both sides has to be selected. This is the case where the indicated (001) and (110) planes in fig. 3 meet each other revealing a net displacement of $1 / 2$ of the (001) and $1 / 2$ of the (110) interplanar spacings. The shift of the (001) lattice fringes is of course observed regardless the chosen variants and this is the component which distinguishes this type (type B) of defect from the former in fig. 1. It has to be mentioned that the atomic configuration at the defect plane is not visible probably due to preferential etching effect during the electropolishing for the TEM sample preparation. The present conclusion only uses the actual positions of related image features on either sides of the defect. 


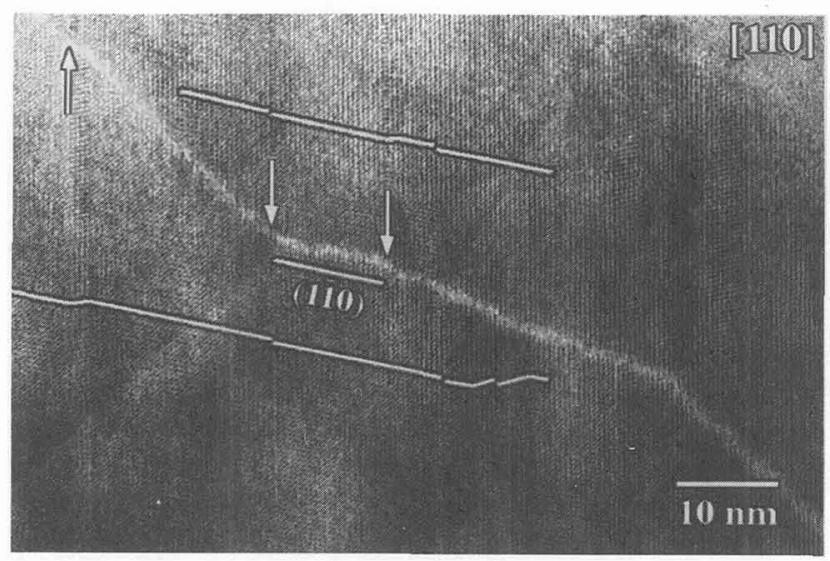

Figure 2: A HRTEM image of a type B planar defect with a zigzag-morphology. The segments which are nearly perpendicular to the twins have a habit plane parallel to the $(1 \overline{1} 0)$ plane of one twin variant.

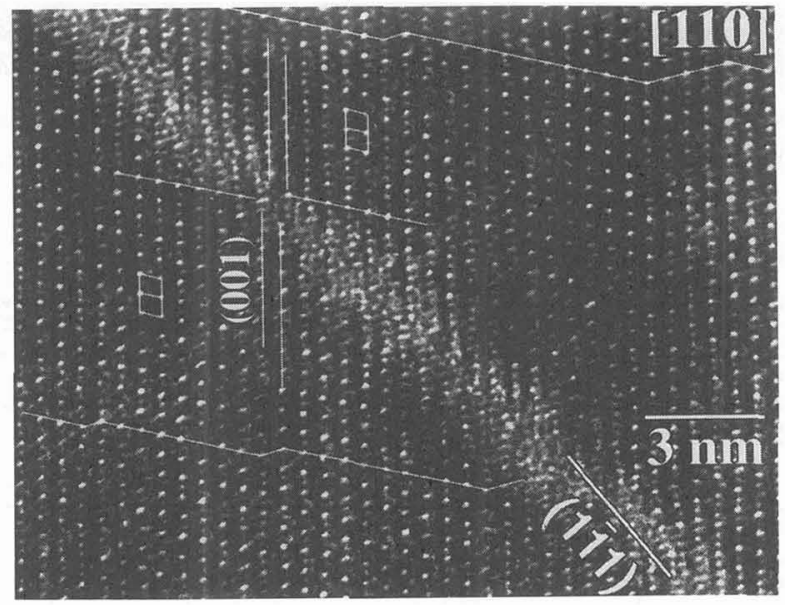

Figure 3: [110] HRTEM image of a type B planar defect with a displacement of $1 / 2$ of $(001)$ and $1 / 2$ of $(1 \overline{10})$ lattice fringes. Microtwins exist with only one unit cell of the variant, the white lines indicate the (110) planes of the variants on both sides of the defect.

In this image microtwins exist and following the (110) lattice fringes (indicated by white zig-zag lines) microtwins can be found which have only one unit cell of the variant and reveal the structure of a stacking fault with a shift of about $1 / 2$ of the (110) lattice fringes. These stacking faults frequently end at the defect as can be seen by comparing the white lines parallel to the (110) planes in the figure, two stacking faults on both sides of the defect are thus clearly not correlated.

In fig. $4 a$ another defect is shown now observed along a [100] zone. In this orientation both martensitic phases yield the same diffraction pattern and can not be distinguished by the image contrast. The defect region is somewhat broader than in the previous cases which could be due to a small inclination or an ill-defined habit plane. A macrotwin with the habit plane (021) is visible at the upper left part of the image. It can be recognized that the defect is almost straight within a given macrotwin and changes the orientation by crossing the macrotwin boundary. The orientation change is not symmetrical to the mirror plane of macrotwin variants. Fig. $4 \mathrm{~b}$ shows an enlargement of the defect, here a displacement of (001) lattice fringes is observed with a shift of $1 / 2$. Due to the displacement of the (001) interplanar spacings this defect is considered as the same defect type of fig. 3. In tab. 1 the observed displacements of the presented defects are listed with respect to the zone axis. 

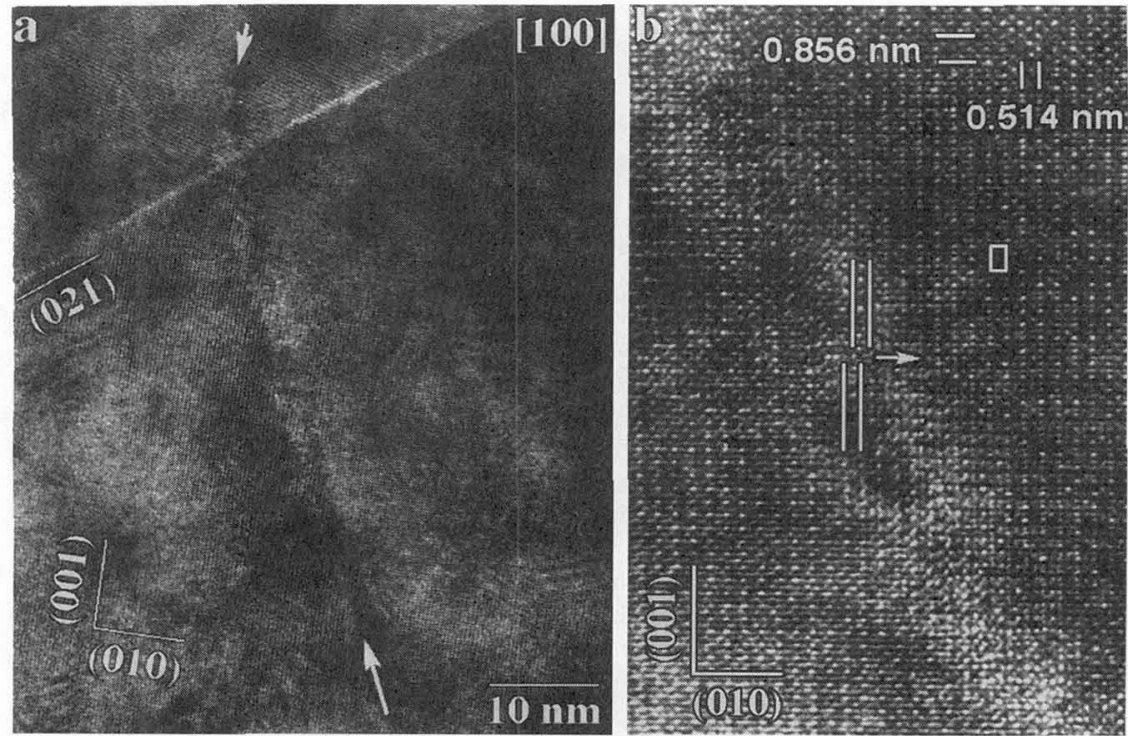

Figure 4: a) [100] HRTEM image of a type B defect in a low magnification. The defect changes its orientation by crossing the macrotwin boundary. b) In a higher magnification a displacement of $1 / 2$ of (001) lattice fringes can be measured whereas no displacement of the $(010)$ fringes is seen.

Table 1: The observed displacements of the different types of planar defects in [110] and [100] zone.

\begin{tabular}{|c|c|c|c|}
\hline & orientation & observed displacement & reference \\
\hline Type A & {$[110]$} & $1 / 2$ of (110) lattice fringes & fig. 1 \\
\hline Type B & {$[110]$} & $1 / 2$ of (110) lattice fringes & fig. 3 \\
& & $1 / 2$ of $(001)$ lattice fringes & \\
\hline Type B & {$[100]$} & $1 / 2$ of $(001)$ lattice fringes & fig. 4 \\
\hline stacking faults & {$[110]$} & $1 / 2$ of (110) lattice fringes & fig. 3 \\
\hline
\end{tabular}

\section{DISCUSSION}

The morphology of the martensite structures in $\mathrm{CuZr}$ is rather complex, not only due to the presence of two martensitic structures but especially because of their mutual configuration and the high density of defects of different types $[7,8]$. However, for the better understanding of the defect structures the crystallographic relationship between the parent B2 phase and the martensite product phases has to be studied more carefully.

As reported before [6,7] the structures of martensitic $\mathrm{CuZr}$ can be obtained by a distortion of the cubic unit cell of the B2 phase to the monoclinic angle and by an additional shuffling of atoms. The lattice correspondence is the same for both martensitic structures:

$[100]_{\mathrm{B} 2} / /[100]_{\mathrm{M}},(011)_{\mathrm{B} 2} / /(001)_{\mathrm{M}}$ and $(011)_{\mathrm{B} 2} / /(010)_{\mathrm{M}}[6,7]$.

According to this structural relationship the structural configuration of the defects in CuZr martensite can be conceived. For example, the mirror planes of the micro and macrotwins in $\mathrm{CuZr}$ martensite which are (001) and (021) planes, respectively, can be correlated with the initial (011) and (010) planes of the B2 phase [8]. The former is the shearing plane of the distortion and the latter the basic plane of the cubic cell.

For the observed defects in CuZr martensite it is reasonable to assume that those defects are formed during the martensitic transformation or are inherited from defects already present in the parent austenite phase. An explanation for the observed defect types A and B can be found assuming an anti-phase boundary in the austenite phase which is created by replacing the $\mathrm{Cu}$ sublattice by $\mathrm{Zr}$ sublattice as shown in the schematic drawing in fig. 5. This anti-phase boundary has in the cubic B2 phase a displacement vector of $\bar{R}=1 / 2[1] 1]_{B 2}$. Due to the crystallographic relationship the $[100]_{B 2}$ axis of $B 2$ becomes the 
$[100]_{\mathrm{MS}}$ axis of the martensitic phases while $[011]_{\mathrm{B} 2}$ axis transforms into the $[010]_{\mathrm{MS}}$ axis. Consequently, the displacement vector in the martensitic phases will be $1 / 2[110]$ for the basic structure and $1 / 4[110]$ for the superstructure, furtheron called $\bar{R}_{A}$. The resulting atomic configuration is presented for the superstructure in fig. 6 in which only $\mathrm{Zr}$ atoms are drawn for clarity. The observed displacement at the defect of the type A in fig. 1 can indeed be accomplished by such a displacement vector which is slightly inclined with respect to the imaging plane of fig. 1 as a result of the monoclinicity. In fig. $6 \mathrm{c}$ the anti-phase boundary is drawn in [110] projection. Here, the unit cell which can be correlated with the imaging code in fig. 1 is drawn with black bold lines and the resulting displacement is in agreement with the shift observed by the defect type A in fig. 1.

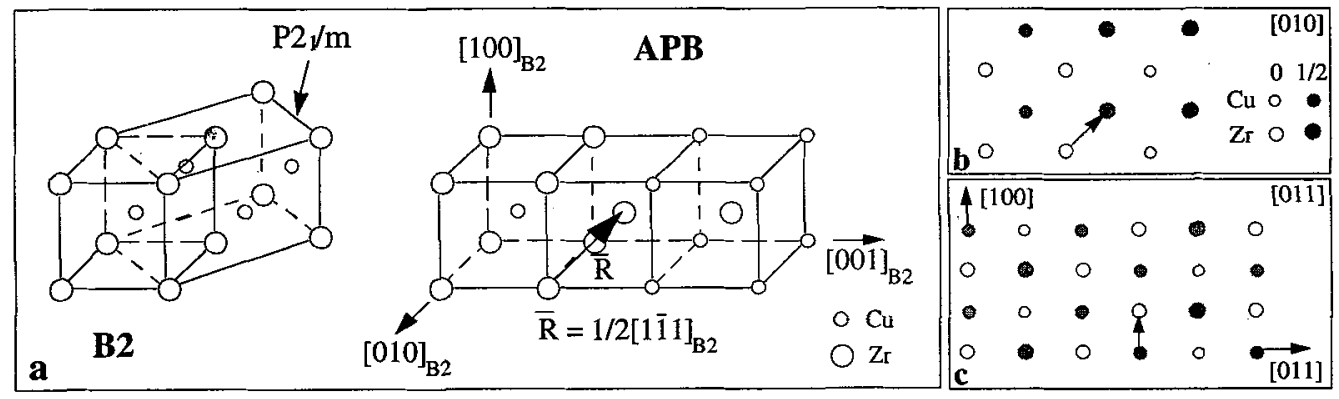

Figure 5: (a) Three-dimensional schematic of the anti-phase boundary with $\overline{\mathrm{R}}=1 / 2[1 \overline{1} 1]_{\mathrm{B} 2}$ in the austenite structure together with two-dimensional projections in (b) [010] and (c) [01 i] zone.

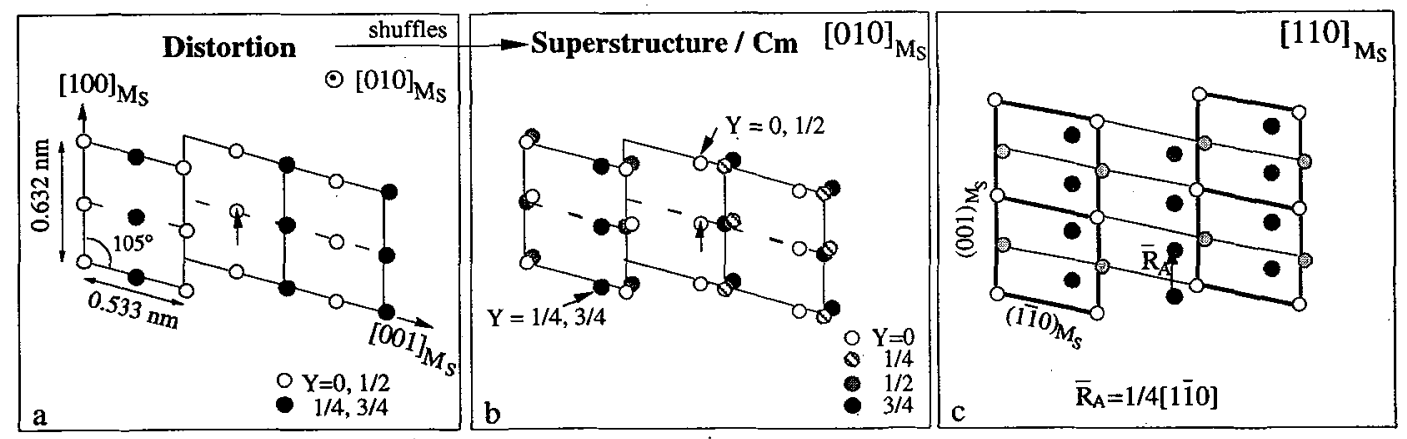

Figure 6: (a) Schematic projection of the displacement at a type A defect in the superstructure including only distortions. For clarity only $\mathrm{Zr}$ atoms are shown. (b) Assuming that the shuffles are not affected by the defect a displacement of $\bar{R}_{A}=1 / 4[110]$ is obtained. c) In [110] projection the displacement vector will result in the complete $1 / 2$ displacement of the (110) atomic planes (cf. fig. 1).

Keeping in mind that the martensitic structure can be considered as the distortion of the B2 unit cell and the subsequent additional shuffles of atoms, there are two different possibilities of shuffling. In the previous discussion on the defect type A the shuffles are not treated separately. This implies that these shuffles are not affected by the defect or, in other words, that the sublattices which are shuffled remain unchanged over the defect. The defects of type B can be understood following the same mechanism but now adding an extra component based on the atomic shuffles. The effect hereof is shown in fig. 7 in which again projections of the superstructure along [010] and [110] directions are shown. Taking the heights of the atoms in [010] direction in fig. 7a into account, an extra component of 1/4[012] is found on top of the one of $1 / 4[110]$ already obtained above. The combination of both yields a final displacement $\bar{R}_{B}=1 / 4[102]$ in the superstructure and $1 / 2[101]$ in the basic one. Indeed, the displacement $\bar{R}_{B}$ results in the observed displacements of the lattice fringes in the HRTEM image of fig. 3 which should be compared with the schematic in fig. $7 \mathrm{~b}$. Moreover the displacement will be observed as a shift of $1 / 2$ of the (001) interplanar spacings in the [100] zone which is in agreement with the observation in fig. $4 \mathrm{~b}$.

As these planar defects appear more or less randomly it is suggested that they are transformed with the matrix together. For stacking faults which have the same well defined habit plane as microtwins it is 
concluded that they are formed like twins after the martensitic transformation, their habit planes are in agreement with the shearing plane of the monoclinic distortion. For the planar defects the habit plane is not well defined and their structures can not be explained only by the distortion or shuffles during the transformation. Moreover the defects are not symmetrical to the microtwin and macrotwin boundaries but they show a strong interaction changing their orientation by crossing these twins. For these behaviours interpretation that the defects were already present before the formation of the twins is more reasonable, keeping in mind that the twins are formed due to the strain relaxation after the transformation. It can be assumed that the initial presence of anti-phase boundaries changes the local strain condition and the twin formation is consequently influenced by the defect structure. This explains the fact that the microtwins are not correlated over the defect (see fig. 3). In the related NiTi system defects with a favoured gliding system was predicted by Kudoh et al. [10]. Their suggestion can be reproduced for the system of CuZr but for the present defect types it fails due to the not well defined habit planes [8].

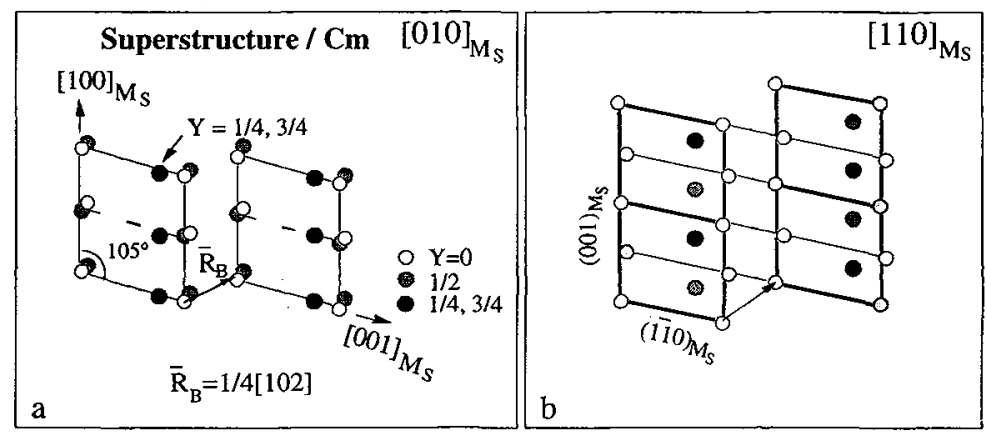

Figure 7: (a) [010] and (b) [110] schematic projections of a type B defect in the superstructure. Shuffles are introduced with different origins on both sides of the defect. The resulting displacement vector is $\overline{\mathrm{R}}_{\mathrm{B}}=1 / 4[102]$. (b) In [110] projection the displacement yields a $1 / 2$ shift of (110) lattice fringes and an additional shift of $1 / 2$ of (001) planes. The unit cell which can be correlated with the imaging code of the HRTEM image in fig. 3 is indicated with black bold lines.

In conclusion, the planar defects in martensitic $\mathrm{CuZr}$ can be explained by the presence of anti-phase boundaries in the parent austenite phase. It has to be pointed out that the anti-phase boundary with the displacement vector $\overline{\mathrm{R}}=1 / 2[1 \overline{1} 1]_{\mathrm{B} 2}$ is the only possible conservative anti-phase configuration in the austenite phase, i.e. not changing the composition. The reason for the two possible defect types in the martensitic phase is the different shuffling of atoms. This result illustrates the microstructural relationship between the austenite and the martensites which is determined by the deformation to the monoclinic angle and the shuffles of atoms.

\section{Acknowledgement}

The authors like to thank Prof. J. Van Humbeeck (KU Leuven) and Dr*. Y. Firstov (Inst. Metal Physics Kiev, Ukraine) for the samples and for fruitful discussions. J.W. Seo thanks the Belgian Programme on Inter University Poles of Attraction (IUAP 46) for financial support.

\section{References}

[1] Arias D. and Abriata J.P., Bulletin of Alloy Phase Diagrams, 11 (1990) 452-459.

[2] Cavalho E.M. and Harris I.R., J. Mater. Sci. 15 (1980) 1224-1230.

[3] Koval Y.N., Firstov G.S. and Kotko A.V., Scripta metall. mater. 27 (1992) 1611-1616.

[4] Zhalko-Titarenko A.V., Yevlashina M.L., Antonov V.N., Yavorskii B.Y., Koval Y.N. and Firstov G.S., Phys. Stat. Sol. (b) 184 (1994) 121-127.

[5] Nicholls A.W., Harris I.R; and Mangen W., J. Mater. Sci. Lett. 5 (1986) 217-220.

[6] Schryvers D., Journal de Physique IV C8 (1995) 1047-1051.

[7] Schryvers D., Firstov G.S., Seo J.W., Humbeeck J.V. and Koval Y.N., Scripta metall. mater. 36 (1997) $1119-1125$.

[8] Seo J.W., Schryvers D., submitted to Acta Met..

[9] Schryvers D., these proceedings (1997).

[10] Kudoh Y., Tokonami M., Miyazaki S. and Otzuka K., Acta Metall 33 (1985) 2049-2056. 